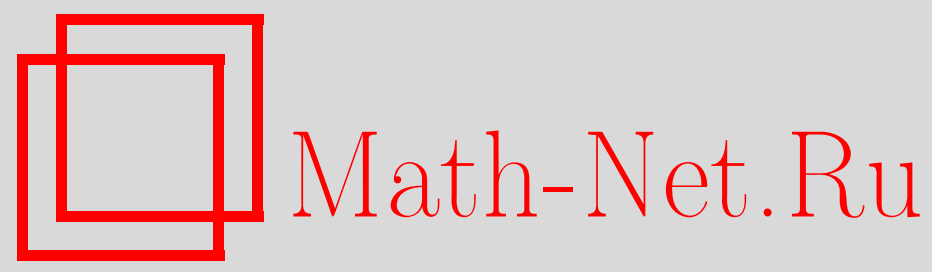

А. В. Арутюнов, Положительность квадратичных форм на пересечении квадрик, Матем. заметки, 2002, том 71, выпуск $1,27-36$

DOI: https://doi.org/10.4213/mzm325

Использование Общероссийского математического портала Math-Net.Ru подразумевает, что вы прочитали и согласны с пользовательским соглашением http://www.mathnet.ru/rus/agreement

Параметры загрузки:

IP : 54.198 .64 .247

26 апреля 2023 г., 09:56:54

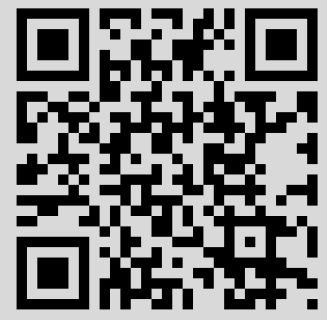




\section{ПОЛОЖИТЕЛЬНОСТЬ КВАДРАТИЧНЫХ ФОРМ НА ПЕРЕСЕЧЕНИИ КВАДРИК}

\section{А. В. Арутюнов}

Исследуется проблема положительной определенности квадратичной формы на конусе, определяемом как множество тех точек, в которых заданные квадратичные формы обращаются в нуль или неотрицательны. Получены необходимые и достаточные условия положительности квадратичной формы на этом конусе. Эти результаты применены к исследованию достаточных условий экстремума второго порядкав анормальных задачах.

Библиограффия: 7 названий.

Пусть даны $(k+1)$ квадратных $n \times n$ симметричных матриц $Q_{i}, i=0, \ldots, k$, определяющих на $\mathbb{R}^{n}$ квадратичные формы $q_{i}: q_{i}(x)=\left\langle Q_{i} x, x\right\rangle$. Требуется получить условия, при которых

$$
\begin{gathered}
q_{0}(x)>0 \quad \forall x: q_{i}(x) \leqslant 0, \quad i=1, \ldots, k_{1}, \\
q_{i}(x)=0, \quad i=k_{1}+1, \ldots, k, \quad x \neq 0,
\end{gathered}
$$

или, иными словами, когда форма $q_{0}$ положительно определена на конусе

$$
K=\left\{x \in \mathbb{R}^{n}: q_{i}(x) \leqslant 0, \quad i=1, \ldots, k_{1}, \quad q_{i}(x)=0, \quad i=k_{1}+1, \ldots, k\right\} .
$$

Здесь $n, k_{1} \leqslant k$ - заданные натуральные числа, причем $k_{1}$ может быть нулем (что соответствует отсутствию ограничений типа неравенств в (1)). Эта задача представляет интерес как сама по себе [1], [2], так и ее применение к теории анормальных экстремальных задач [3] и к некоторым прикладным задачам (библиографию см. в [4]). В этой заметке содержится вьвод соответствующих условий и их приложение к теории анормальных экстремальных задач.

1. Критерий положительности квадратичной формы. Очевидно, что условие (1) равносильно тому, что в задаче минимизации

$$
q_{0}(x) \rightarrow \min , \quad q_{i}(x) \leqslant 0, \quad i=1, \ldots, k_{1}, \quad q_{i}(x)=0, \quad i=k_{1}+1, \ldots, k,
$$

точка $x_{*}=0$ является строгим локальным минимумом. Эта точка является анормальной (см. [3]) в рассматриваемой задаче. Применим к ней необходимые условия второго порядка из [3], [5], справедливые для анормальных задач.

Работа выполнена при поддержке Российского фонда фундаментальных исследований, грант № 99-01-01135. 
Для целого $r \geqslant 0$ через $\Lambda_{r}$ обозначим множество $(k+1)$-мерных векторов $\lambda=\left(\lambda_{0}, \lambda_{1}\right.$, $\left.\ldots, \lambda_{k}\right)$, для которых

$$
\lambda_{i} \geqslant 0, \quad i=0, \ldots, k_{1}, \quad|\lambda|=1, \quad \operatorname{ind}(\lambda q) \leqslant r .
$$

Здесь $q=\left(q_{0}, \ldots, q_{k}\right), \lambda q=\sum_{i=0}^{k} \lambda_{i} q_{i}$, a ind это (отрищательньй) индекс квадратичной формы (равный количеству отрищательных собственных значений определяющей ее симметричной матрицы). Тогда множество $\Lambda_{k-1}$ непусто и

$$
\max _{\lambda \in \Lambda_{k-1}}\langle\lambda, q(x)\rangle \geqslant 0 \quad \forall x \in \mathbb{R}^{n}
$$

Если ограничения типа неравенств отсутствуют, т.е. $k_{1}=0$, то $(2)$ - это просто теорема 2 из [5]. (В общем случае она доказывается с использованием схемы из [3, с. 30-35] аналогично.)

Однако среди векторов $\lambda$, принадлежащих $\Lambda_{k-1}$, могут оказаться и те, для которых $\lambda_{0}=0$. А это приводит к тому, что условия (2) перестают зависеть от формы $q_{0}$, что может сделать их неинформативньми. Так обязательно произойдет, если conv $\Lambda_{k-1}-$ выпуклая оболочка множества $\Lambda_{k-1}$ - содержит нуль, так как в этом случае, очевидно, (2) выполняется автоматически. Вот соответствующий пример: $k=2, k_{1}=0$, $q_{1}(x)=x_{1} x_{2}$, а формы $q_{0}$ и $q_{2}$ произвольны. Очевидно, $\Lambda_{1}$ содержит отрезок $\left(0, \lambda_{1}, 0\right)$, $\left|\lambda_{1}\right| \leqslant 1$, и значит $(2)$ выполняется для любых $q_{0}, q_{2}$ и, в частности, для которых $(1)$ нарушается (например для $q_{0}(x)=-|x|^{2}, q_{2}=0$ ).

Наша цель - доказать, что если форма $q_{0}$ положительна на конусе $K$, то в $(2)$ можно ограничиться лишь теми $\lambda \in \Lambda_{k-1}$, для которых $\lambda_{0}>0$. Это позволяет усилить необходимые условия (2) до достаточных.

Положим

$$
\Lambda_{r}^{+}=\left\{\lambda \in \Lambda_{r}: \lambda_{0}>0\right\} .
$$

Теорема 1. Для того чтобы форма qо была положительно определена на конусе $K$, необходимо и достаточно, чтобь мнохество $\Lambda_{k-1}^{+}$был непусто и выполнялось

$$
\forall x \neq 0 \quad \exists \lambda=\lambda(x) \in \Lambda_{k-1}^{+}:\langle\lambda, q(x)\rangle>0 .
$$

ДокАЗАТЕЛЬСтво. Достаточность очевидна. Докажем необходимость. В силу (1) существует такое $\varepsilon>0$, что для квадратичной формы $q_{0}^{\varepsilon}$, определенной по формуле $q_{0}^{\varepsilon}(x)=q_{0}(x)-\varepsilon|x|^{2}$, имеет место

$$
q_{0}^{\varepsilon}(x) \geqslant \varepsilon|x|^{2} \quad \forall x \in K
$$

Зафиксируем произвольное $h \in \mathbb{R}^{n}, h \neq 0$. Рассмотрим задачу минимизации

$$
\left\{\begin{array}{l}
q_{0}^{\varepsilon}(x)-\chi q_{0}^{\varepsilon}(h) \rightarrow \min , \\
q_{i}(x)-\chi q_{i}(h) \leqslant 0, \quad i=1, \ldots, k_{1}, \\
q_{i}(x)-\chi q_{i}(h)=0, \quad i=k_{1}+1, \ldots, k, \quad 0 \leqslant \chi \leqslant 1 .
\end{array}\right.
$$

Минимум в этой задаче ищется по переменным $(x, \chi) \in \mathbb{R}^{n} \times \mathbb{R}^{1}$. Из (4) легко вытекает, что инфимум в ней конечен и достигается. Среди точек минимума $(\bar{x}, \bar{\chi})$ найдется такая, 
что $\bar{\chi}>0$. (Действительно, если в точке минимума $\bar{\chi}=0$, то в силу (4) $\bar{x}=0$ и, следовательно, точка $x=h, \chi=1$ также реализует минимум.)

Итак, возьмем точку минимума $(\bar{x}, \bar{\chi})$, в которой $\bar{\chi}>0$. Рассмотрим семейство задач минимизации, зависящее от натурального параметра $j$ :

$$
f_{j}(x, \chi) \rightarrow \min , \quad 0 \leqslant \chi \leqslant 1
$$

где

$$
\begin{aligned}
f_{j}(x, \chi)= & q_{0}^{\varepsilon}(x)-\chi q_{0}^{\varepsilon}(h) \\
& +j\left(\sum_{i=1}^{k_{1}} \max \left(q_{i}(x)-\chi q_{i}(h), 0\right)^{4}+\sum_{i=k_{1}+1}^{k}\left(q_{i}(x)-\chi q_{i}(h)\right)^{4}\right)+(\chi-\bar{\chi})^{2} .
\end{aligned}
$$

Задачи этого семейства назовем $j$-задачами.

При каждом $j$ минимум в $j$-задаче достигается. Докажем это. Действительно, предположим противное. Тогда для $j$-задачи существует такая минимизирующая последовательность $\left\{x_{l}, \chi_{l}\right\}$, что

$$
f_{j}\left(x_{l}, \chi_{l}\right)<0 \quad \forall l, \quad\left|x_{l}\right| \rightarrow \infty, \quad\left|x_{l}\right|^{-1} x_{l} \rightarrow z, \quad l \rightarrow \infty
$$

для некоторого единичного вектора $z$. Деля выражение для $f_{j}$ на $\left|x_{l}\right|^{4}$, используя ограниченность последовательности $\left\{\chi_{l}\right\}$ и переходя к пределу при $l \rightarrow \infty$, получаем

$$
j\left(\sum_{i=1}^{k_{1}} \max \left(q_{i}(z), 0\right)^{4}+\sum_{i=k_{1}+1}^{k} q_{i}(z)^{4}\right) \leqslant 0 \Longrightarrow z \in K
$$

Поэтому в силу (4) $\exists \delta>0: q_{0}^{\varepsilon}\left(\left|x_{l}\right|^{-1} x_{l}\right) \geqslant \delta$ при больших $l$. Деля выражение для $f_{j}$ на $\left|x_{l}\right|$, получаем, что $f_{j}\left(x_{l}, \chi_{l}\right)>0$ при больших $l$. Это противоречие доказывает нужное утверждение.

Таким образом, минимум в $j$-задаче достигается в некоторой точке $\left(x_{j}, \chi_{j}\right)$. Более того, аналогично доказьвается (это стандартное рассуждение метода возмущений (см. [3, c. 31])), что $x_{j} \rightarrow 0, \chi_{j} \rightarrow \bar{\chi}, j \rightarrow \infty$.

Выберем такое достаточно большое $\bar{j}$, что

$$
\chi_{\bar{j}}>0, \quad 2\left|\chi_{\bar{j}}-\bar{\chi}\right|<\varepsilon|h|^{2} .
$$

При $j=\bar{j}$ вьпишем в рассматриваемой задаче необходимые условия минимума первого и второго порядка в точке $\left(x_{j}, \chi_{j}\right)$. Положим $f=f_{\bar{j}}$. Учитьвая, что $\chi_{j}>0$, имеем

$$
\frac{\partial f}{\partial \chi}\left(x_{j}, \chi_{j}\right)=-q_{0}^{\varepsilon}(h)-\sum_{i=1}^{k} \lambda_{i} q_{i}(h)+2\left(\chi_{j}-\bar{\chi}\right) \leqslant 0
$$


откуда

$$
\begin{gathered}
q_{0}^{\varepsilon}(h)+\sum_{i=1}^{k} \lambda_{i} q_{i}(h) \geqslant-2\left|\chi_{j}-\bar{\chi}\right|, \\
\frac{\partial f}{\partial x}\left(x_{j}, \chi_{j}\right)=2\left(Q_{0}^{\varepsilon}+\sum_{i=1}^{k} \lambda_{i} Q_{i}\right) x_{j}=0, \\
\frac{\partial^{2} f}{\partial x^{2}}\left(x_{j}, \chi_{j}\right)[\xi, \xi]=2\left(Q_{0}^{\varepsilon}+\sum_{i=1}^{k} \lambda_{i} Q_{i}\right)[\xi, \xi] \\
+12 j\left(\sum_{i=1}^{k_{1}} \max \left(q_{i}\left(x_{j}\right)-\chi_{j} q_{i}(h), 0\right)^{2}\left\langle Q_{i} x_{j}, \xi\right\rangle^{2}\right. \\
\left.+\sum_{i=k_{1}+1}^{k}\left(q_{i}\left(x_{j}\right)-\chi_{j} q_{i}(h)\right)^{2}\left\langle Q_{i} x_{j}, \xi\right\rangle^{2}\right) \geqslant 0 \quad \forall \xi
\end{gathered}
$$

где

$$
\begin{gathered}
\lambda_{i}=4 \max \left(q_{i}\left(x_{j}\right)-\chi_{j} q_{i}(h), 0\right)^{3} \geqslant 0, \quad i=1, \ldots, k_{1}, \\
\lambda_{i}=4\left(q_{i}\left(x_{j}\right)-\chi_{j} q_{i}(h)\right)^{3}, \quad i=k_{1}+1, \ldots, k, \quad Q_{0}^{\varepsilon}=Q_{0}-\varepsilon E
\end{gathered}
$$

а $E$ - единичная матрица.

Введем в рассмотрение подпространство

$$
\widetilde{\Pi}=\left\{\xi:\left\langle Q_{i} x_{j}, \xi\right\rangle=0, \quad i=1, \ldots, k\right\}
$$

и рассмотрим два случая.

Пусть вначале $x_{j} \in \widetilde{\Pi}$. Тогда

$$
\left\langle Q_{i} x_{j}, x_{j}\right\rangle=0, \quad i=1, \ldots, k \Longrightarrow x_{j} \in K
$$

Умножая скалярно равенство $(7)$ на вектор $x_{j}$, получаем $q_{0}^{\varepsilon}\left(x_{j}\right)=0$, откуда в силу (4)

$$
x_{j}=0 \Longrightarrow Q_{i} x_{j}=0, \quad i=1, \ldots, k,
$$

и, следовательно, в силу (8) квадратичная форма $q_{0}^{\varepsilon}$ неотрицательно определена. Поэтому (3) выполняется при $\lambda=(1,0, \ldots, 0)$.

Рассмотрим второй случай: $x_{j} \notin \widetilde{\Pi}$. Возьмем вектор

$$
\lambda=|\widehat{\lambda}|^{-1} \widehat{\lambda}, \quad \text { где } \widehat{\lambda}=\left(1, \lambda_{1}, \ldots, \lambda_{k}\right) .
$$

Из неравенств (5), (6) и определения формы $q_{0}^{\varepsilon}$ вытекает, что $\langle\lambda, q(h)\rangle>0$. Для доказательства (3) нам осталось показать, что $\lambda \in \Lambda_{k-1}$ (ведь $\lambda_{0}>0$ по построению). Сделаем это. 
Снова рассмотрим подпространство ПГ. Его коразмерность, очевидно, не превышает $k$ и в силу (8) квадратичная форма

$$
\bar{q}=q_{0}^{\varepsilon}+\sum_{i=1}^{k} \lambda_{i} q_{i}
$$

неотрицательно определена на этом подпространстве. Обозначим через П алгебраическую сумму подпространства $\widetilde{\Pi}$ и линейной оболочки вектора $x_{j}$. Тогда коразмерность подпространства П непревышает $k-1$, так как по сделанному предположению $x_{j} \notin \widetilde{\Pi}$. В то же время форма $\bar{q}$ неотрицательна на этом же подпространстве П, так как она неотрицательна на $\widetilde{\Pi}$, и в силу (7) матрица этой формы аннулирует вектор $x_{j}$. Следовательно, $\lambda \in \Lambda_{k-1}$. Теорема доказана.

Сразу же отметим, что теорема 1 является весьма далеким развитием теоремы Финслера (см., например, [4]), которая соответствует случаю $k=1$. Для $k \leqslant 2$ близкие вопросы изучены в [5] (там же имеется библиография).

Обсудим полученный результат.

ЗАМЕЧАНИЕ 1. Для $\varepsilon>0$ положим

$$
\Lambda_{r}^{\varepsilon}=\left\{\lambda \in \Lambda_{r}: \lambda_{0} \geqslant \varepsilon\right\}
$$

Если форма $q_{0}$ положительно определена на $K$, то существует такое $\varepsilon>0$, что

$$
\max _{\lambda \in \Lambda_{k-1}^{\varepsilon}}\langle\lambda, q(x)\rangle \geqslant \varepsilon|x|^{2} \quad \forall x \in \mathbb{R}^{n} \text {. }
$$

Докажем это. Пусть $S$ - единичная сфера из $\mathbb{R}^{n}$. Возьмем произвольньй вектор $\bar{x} \in S$. По теореме 1 для него существует такое $\lambda(\bar{x}) \in \Lambda_{k-1}^{+}$, что $\langle\lambda(\bar{x}), q(\bar{x})\rangle>0$. Поэтому для всех $x \in S$ из некоторой окрестности $O(\bar{x})$ точки $\bar{x}$ имеет место

$$
\langle\lambda(\bar{x}), q(x)\rangle>\frac{1}{2}\langle\lambda(\bar{x}), q(\bar{x})\rangle .
$$

Покроем компакт $S$ такими окрестностями. Выбирая из них конечное подпокрытие,найдем такие $x_{j} \in S, j=1, \ldots, j_{1}$, что объединение $O\left(x_{j}\right)$ по всем $j$ покрывает $S$. Остается взять

$$
\varepsilon=\min \left\{\lambda_{0}\left(h_{j}\right), j=1, \ldots, j_{1}, \quad \frac{1}{2}\left\langle\lambda\left(x_{j}\right), q\left(x_{j}\right)\right\rangle\right\} .
$$

Ясно, что построенное число $\varepsilon>0$ является искомым.

ЗАмЕчАнИЕ 2. Необходимость в теореме 1 нельзя обобщить на случай, когда форма $q_{0}$ всего лишь неотрицательно определена на конусе $K$, даже заменив в (3) строгое неравенство на нестрогое. Дело в том, что если $q_{0}$ неотрицательна на $K$, то множество $\Lambda_{k-1}^{+}$тем не менее может оказаться пустым. Вот соответствующий простой пример:

$$
k=1, \quad k_{1}=0, \quad n=2, \quad q_{0}(x)=x_{1} x_{2}, \quad q_{1}(x)=x_{1}^{2} .
$$

Очевидно, что $q_{0}(x)=0 \forall x: q_{1}(x)=0$, однако $\Lambda_{0}^{+}=\varnothing$. 
В этом примере форма $q_{1}$ неотрицательна. Приведем менее тривиальньй пример, в котором никакая нетривиальная линейная комбинация форм $q_{i}$ не является знакоопределенной. Пусть

$$
\begin{gathered}
k=3, \quad k_{1}=0, \quad n=2 m, \quad m>4, \quad q_{0}(x)=\sum_{j=1}^{m} x_{j} x_{j+m}, \\
q_{1}(x)=x_{1} x_{2}, \quad q_{2}(x)=x_{1}^{2}-x_{2}^{2}, \quad q_{3}(x)=-x_{1}^{2}-x_{2}^{2}+x_{3}^{2}+\cdots+x_{m}^{2} .
\end{gathered}
$$

Здесь также $q_{0}(x)=0 \forall x \in K$, однако множество $\Lambda_{2}^{+}$пусто.

ЗАмЕчАниЕ 3 . K сожалению, утверждение теоремы 1 существенно конечномерно. А именно, необходимость в ней нельзя обобщить на случай, когда формы $q_{i}$ определены на бесконечномерном пространстве. Вот соответствующий простой пример. Пусть $X$ - гильбертово пространство, $A: X \rightarrow X$-заданный линейный, симметричный, компактньй, положительно определенньй оператор, $q_{0}(x)=-|x|^{2}, q_{1}(x)=\langle A x, x\rangle$. Тогда конус $\left\{x: q_{1}(x)=0\right\}$ нулевой и, значит, форма $q_{0}$ положительна на нем. В то же время множество $\Lambda_{0}^{+}$пусто, так как индекс формы $q_{0}+\lambda_{1} q_{1}$ бесконечен для любого $\lambda_{1}$.

Зададимся таким вопросом. Когда система квадратичных равенств и неравенств

$$
q_{i}(x) \leqslant 0, \quad i=1, \ldots, k_{1}, \quad q_{i}(x)=0, \quad i=k_{1}+1, \ldots, k,
$$

имеет ненулевое решение? Ответ на него таков. Система (9) имеет ненулевое решение тогда и только тогда, когда найдется такой ненулевой вектор $h \in \mathbb{R}^{n}$, что

$$
\sum_{i=1}^{k} \lambda_{i} q_{i}(h) \leqslant 0 \quad \forall \lambda=\left(\lambda_{1}, \cdots, \lambda_{k}\right): \lambda_{i} \geqslant 0, \quad i=1, \ldots, k_{1}, \quad \text { ind }\left(\sum_{i=1}^{k} \lambda_{i} q_{i}\right) \leqslant k-1 .
$$

Докажем это. Действительно, отсутствие ненулевых решений у (9) равносильно тому, что

$$
\begin{gathered}
q_{0}(x)=-|x|^{2}>0 \quad \forall x: q_{i}(x) \leqslant 0, \quad i=1, \ldots, k_{1} \\
q_{i}(x)=0, \quad i=k_{1}+1, \ldots, k, \quad x \neq 0 .
\end{gathered}
$$

Теперь осталось применить теорему 1.

2. Приложение к теории экстремальных задач. Теорема 1 гарантирует, что в задаче на строгий минимум квадратичного функционала при квадратичных ограничениях в условиях экстремума для анормальных задач (см. [3, гл. 1, п. 1.4]) $\lambda_{0}$ (множитель Лагранжа при минимизируемом функционале) всегда можно брать равным единице. Выясним на какие классы задач, не являющихся квадратичными, распространяется это утверждение.

Рассмотрим гладкую нелинейную задачу минимизации:

$$
f_{0}(x) \rightarrow \min , \quad F(x)=0 .
$$

Здесь заданное отображение $F=\left(f_{1}, \ldots, f_{k}\right): \mathbb{R}^{n} \rightarrow \mathbb{R}^{k}$ и функция $f_{0}$ предполагаются дважды непрерывно дифференцируемыми в окрестности точки $x_{*} \in \mathbb{R}^{n}: F\left(x_{*}\right)=0$. Сформулируем необходимые условия экстремума в точке $x_{*}$ (см. [3, глава 1, п. 4.1$]$ ). 
Рассмотрим функцию Лагранжа

$$
\mathscr{L}(x, \lambda)=\sum_{i=0}^{k} \lambda_{i} f_{i}(x), \quad \lambda=\left(\lambda_{0}, \lambda_{1}, \ldots, \lambda_{k}\right)
$$

Обозначим через $\Lambda\left(x_{*}\right)$ множество нормированных множителей Лагранжа $\lambda$, отвечающих ей в силу правила множителей Лагранжа:

$$
\frac{\partial \mathscr{L}}{\partial x}\left(x_{*}, \lambda\right)=0, \quad \lambda_{0} \geqslant 0, \quad|\lambda|=1 .
$$

Для натурального $r$ введем в рассмотрение множество, состоящее из тех множителей Лагранжа $\lambda \in \Lambda\left(x_{*}\right)$, для которых существует такое (зависящее от $\lambda$ ) подпространство $X \subseteq \mathbb{R}^{n}$, что

$$
\operatorname{codim} X \leqslant r, \quad X \subseteq \operatorname{Ker} \frac{\partial F}{\partial x}\left(x_{*}\right), \quad \frac{\partial^{2} \mathscr{L}}{\partial x^{2}}\left(x_{*}, \lambda\right)[x, x] \geqslant 0 \quad \forall x \in \mathbb{R}^{n} .
$$

Это множество множителей Лагранжа будем обозначать через $\Lambda_{r}\left(x_{*}\right)$.

В [3, гл. 1 , теорема 4.1$]$ доказано, что если в рассматриваемой задаче точка $x_{*}$ является локальньм минимумом, то множество $\Lambda_{k}\left(x_{*}\right)$ непусто и, более того,

$$
\max _{\lambda \in \Lambda_{k}\left(x_{*}\right)} \frac{\partial^{2} \mathscr{L}}{\partial x^{2}}\left(x_{*}, \lambda\right)[x, x] \geqslant 0 \quad \forall x \in \operatorname{Ker} F^{\prime}\left(x_{*}\right) .
$$

Итак, спрашивается, если точка $x_{*}$ является локальным минимумом, то когда можно гарантировать, что в сформулированных условиях экстремума можно обойтись лишь теми множителями Лагранжа $\lambda$, для которых $\lambda_{0}>0$ ? Если точка $x_{*}$ нормальна, т.е. оператор $\operatorname{Im} F^{\prime}\left(x_{*}\right)$ сюръективен $\left(\operatorname{Im} F^{\prime}\left(x_{*}\right)=\mathbb{R}^{k}\right)$, то, как известно, ответ утвердительный. Если же точка $x_{*}$ анормальна (т.е. оператор $\operatorname{Im} F^{\prime}\left(x_{*}\right)$ сюръективньп не является), то, вообще говоря, это уже не так. (Например, если $f_{0}$ ненулевая линейная функция и $F(x)=|x|^{2}$, то $\lambda_{0}=0 \forall \lambda \in \Lambda(0)$.)

Однако теорема 1 позволяет выделить широкий класс задач, для которых ответ на поставленньй вопрос также является утвердительным. Введем требуемое определение.

Для произвольного $h \in \mathbb{R}^{n}$ определим линейное отображение $G(h): \mathbb{R}^{n} \rightarrow \mathbb{R}^{k}$ формулой

$$
G(h) x=F^{\prime}\left(x_{*}\right) x+\pi F^{\prime \prime}\left(x_{*}\right)[h, x],
$$

где $\pi$ - оператор ортогонального проектирования $\mathbb{R}^{k}$ на подпространство $\left(\operatorname{Im} F^{\prime}\left(x_{*}\right)\right)^{\perp}$. Введем в рассмотрение конус

$$
\mathscr{K}=\left\{h \in \mathbb{R}^{n}: F^{\prime}\left(x_{*}\right) h=0, \quad \pi F^{\prime \prime}\left(x_{*}\right)[h, h]=0\right\} .
$$

ОПРЕДЕЛЕНИЕ (см. [6]). Отображение $F$ назовем 2-регулярным в точке $x_{*}: F\left(x_{*}\right)$ $=0$ nо направлению $h \in \mathscr{K}$, если оператор $G(h)$ сюръективен. Отображение $F$ назовем 2-регулярным в точке $x_{*}$, если оно 2-регулярно в ней по любому ненулевому направлению $h \in \mathscr{K}$. 
ПРЕДЛОЖЕНИЕ. Предположим, что точка $x_{*}$ анормальна (m.e. $\left.\operatorname{Im} F^{\prime}\left(x_{*}\right) \neq \mathbb{R}^{k}\right)$, отобрахсение F 2-регулярно в ней,

$$
f^{\prime}\left(x_{*}\right) \in \operatorname{Im}\left(F^{\prime}\left(x_{*}\right)\right)^{*}
$$

$u$ существует такое $\delta>0$, что $f_{0}(x) \geqslant f_{0}\left(x_{*}\right)+\delta\left|x-x_{*}\right|^{2}$ для всех $x: F(x)=0$, лехащих в некоторой окрестности точки $x_{*}$.

Тогда существует такое $\varepsilon>0$, что

$$
\max _{\lambda \in \Lambda_{k-1}^{\varepsilon}\left(x_{*}\right)} \frac{\partial^{2} \mathscr{L}}{\partial x^{2}}\left(x_{*}, \lambda\right)[x, x] \geqslant 0 \quad \forall x \in \operatorname{Ker} F^{\prime}\left(x_{*}\right),
$$

əде $\Lambda_{r}^{\varepsilon}\left(x_{*}\right)=\left\{\lambda \in \Lambda_{r}\left(x_{*}\right): \lambda_{0} \geqslant \varepsilon\right\}$.

ДокАЗАтЕльство. В силу (10) будем, не теряя общности, считать, что $f^{\prime}\left(x_{*}\right)=0$. По теореме 1 из [6] в силу 2-регулярности отображения $F$ в точке $x_{*}$ получаем, что в ее окрестности множество $\{x: F(x)=0\}$ локально диффеоморфно конусу $\mathscr{K}$ в окрестности нуля. Отсюда, используя последнее предположение леммы, получаем, что

$$
f_{0}^{\prime \prime}\left(x_{*}\right)[x, x]>0 \quad \forall x \in \mathscr{K}: x \neq 0 .
$$

Теперь остается применить теорему 1 и замечание 1 к ней. Предложение доказано.

ЗАмЕчАнИЕ. В формулировке предложения предположение о 2-регулярности отображения $F$ в точке $x_{*}$ опустить нельзя. Это показьвает следующий простой пример: $-|x|^{2} \rightarrow \min ,|x|^{3}=0$. В нем минимум достигается в нуле, однако $\lambda_{0}=0 \forall \lambda \in \Lambda_{k-1}(0)$.

Перейдем к достаточным условиям экстремума второго порядка. Для конечномерного случая, который мы только и рассматриваем, они имеют следующий вид (см. [7, $\S 8],[3, \S 1.7])$. Если

$$
\max _{\lambda \in \Lambda\left(x_{*}\right)} \frac{\partial^{2} \mathscr{L}}{\partial x^{2}}\left(x_{*}, \lambda\right)[x, x]>0 \quad \forall x \in \operatorname{Ker} F^{\prime}\left(x_{*}\right): x \neq 0,
$$

то $x_{*}$ является точкой строго локального минимума в рассматриваемой задаче.

Сравнивая достаточные условия с выписанными вьше необходимьми, видим, что в них фигурируют разные множества множителей Лагранжа: $\Lambda\left(x_{*}\right)$ и $\Lambda_{k}\left(x_{*}\right)$. Это несоответствие представляется неестественным. В то же время менять в необходимых условиях множество $\Lambda_{k}\left(x_{*}\right)$ на большее $-\Lambda\left(x_{*}\right)$ - нецелесообразно, так как в этом случае ослабление необходимых условий приводит к тому, что они становятся тривиальными (т.е. вьполняются автоматически) для любых анормальных задач (см. [3, с. 27], [7, c. 105]).

В связи с этим зададимся вопросом: следует ли заменить в достаточных условиях множество $\Lambda\left(x_{*}\right)$ на меньшее $-\Lambda_{k}\left(x_{*}\right)$ ? Формально достаточные условия при этом ослабляются, но, как показьвает следующая теорема, дающая на поставленньй вопрос утвердительньй ответ, на самом деле это не так.

ТЕоремА 2. Предположим, что точка $x_{*}$ анормальна и выполняется условие (11). Тогда имеет место

$$
\max _{\lambda \in \Lambda_{k-1}\left(x_{*}\right)} \frac{\partial^{2} \mathscr{L}}{\partial x^{2}}\left(x_{*}, \lambda\right)[x, x]>0 \quad \forall x \in \operatorname{Ker} F^{\prime}\left(x_{*}\right): x \neq 0 .
$$


ДокАЗАТЕльСтво. Докажем существование такого $\bar{\lambda} \in \Lambda\left(x_{*}\right)$, что $\bar{\lambda}_{0}>0$ и квадратичная форма

$$
\frac{\partial^{2} \mathscr{L}}{\partial x^{2}}\left(x_{*}, \bar{\lambda}\right)
$$

положительно определена на конусе $\mathscr{K}$. При этом будем предполагать, что конус $\mathscr{K}$ ненулевой (в противном случае искомое утверждение выполняется автоматически).

Предположим вначале, что $f_{0}^{\prime}\left(x_{*}\right)=0$. Покажем, что тогда вектор $\bar{\lambda}=(1,0, \ldots, 0)$ является искомым. Действительно, возьмем произвольньй $x \in \mathscr{K}, x \neq 0$. В силу (11)

$$
\exists \lambda \in \Lambda\left(x_{*}\right): \frac{\partial^{2} \mathscr{L}}{\partial x^{2}}\left(x_{*}, \lambda\right)[x, x]>0 .
$$

Положим $\mu=\left(\lambda_{1}, \ldots, \lambda_{k}\right)$. Тогда из равенств

$$
f_{0}^{\prime}\left(x_{*}\right)=0, \quad \frac{\partial \mathscr{L}}{\partial x}\left(x_{*}, \lambda\right)=0
$$

имеем $\mu \pi=\mu$, откуда

$$
\left\langle\mu, F^{\prime \prime}\left(x_{*}\right)[x, x]\right\rangle=\left\langle\mu \pi, F^{\prime \prime}\left(x_{*}\right)[x, x]\right\rangle=0,
$$

так как $x \in \mathscr{K}$. Теперь из равенства

$$
\left\langle\mu, F^{\prime \prime}\left(x_{*}\right)[x, x]\right\rangle=0
$$

в силу (11) получаем $f^{\prime \prime}\left(x_{*}\right)[x, x]>0$, что и требовалось доказать.

Сведем теперь случай произвольного $f_{0}^{\prime}\left(x_{*}\right)$ к рассмотренному. Выберем какой-нибудь вектор $h \in \mathscr{K}, h \neq 0$. В силу (11)

$$
\exists \bar{\lambda} \in \Lambda\left(x_{*}\right): \frac{\partial^{2} \mathscr{L}}{\partial x^{2}}\left(x_{*}, \bar{\lambda}\right)[h, h]>0 .
$$

Покажем, что $\bar{\lambda}_{0}>0$. Действительно, предположим противное. Тогда, обозначив $\mu=\left(\bar{\lambda}_{1}, \ldots, \bar{\lambda}_{k}\right)$, имеем

$$
\mu \neq 0, \quad \mu F^{\prime}\left(x_{*}\right)^{*}=0 \Longrightarrow \mu \pi=\mu
$$

откуда в силу (11)

$$
0<\left\langle\mu, F^{\prime \prime}\left(x_{*}\right)[h, h]\right\rangle=\left\langle\mu \pi, F^{\prime \prime}\left(x_{*}\right)[h, h]\right\rangle=0,
$$

так как $h \in \mathscr{K}$. Полученное противоречие доказывает, что $\bar{\lambda}_{0}>0$.

Теперь рассмотрим задачу минимизации

$$
\mathscr{L}(x, \bar{\lambda}) \rightarrow \min , \quad F(x)=0 .
$$

Для нее также вьполнены достаточные условия второго порядка (11). Поэтому, заменяя функцию $f_{0}(\cdot)$ на $\mathscr{L}(\cdot, \bar{\lambda})$ и учитывая, что $\partial \mathscr{L} / \partial x\left(x_{*}, \bar{\lambda}\right)=0$, приходим к случаю, рассмотренному вьше. 
Таким образом, доказано, что квадратичная форма

$$
q_{0}=\frac{\partial^{2} \mathscr{L}}{\partial x^{2}}\left(x_{*}, \bar{\lambda}\right)
$$

положительно определена на конусе $\mathscr{K}$, которьй задан на подпространстве $\operatorname{Ker} F^{\prime}\left(x_{*}\right)$ системой квадратичных равенств: $q_{i}(x)=0, i=1, \ldots, \tilde{k}$, для некоторого $\tilde{k} \leqslant k$. Здесь $q_{i}$ - некоторые линейные комбинации квадратичных форм $f_{i}^{\prime \prime}\left(x_{*}\right)$. Применяя теперь теорему 1 и учитьвая, что $\bar{\lambda}_{0}>0$, получаем (12). Теорема доказана.

Теорема 2 показьвает, что достаточные условия второго порядка естественно брать в виде

$$
\max _{\lambda \in \Lambda_{k}\left(x_{*}\right)} \frac{\partial^{2} \mathscr{L}}{\partial x^{2}}\left(x_{*}, \lambda\right)[x, x]>0 \quad \forall x \in \operatorname{Ker} F^{\prime}\left(x_{*}\right): x \neq 0,
$$

не ослабляя их и не нарушая их гармоническую связь с необходимыми условиями. В заключение отметим, что полученные результаты справедливы также и для экстремальных задач, содержащих ограничения типа неравенств.

\section{СПИСОК ЦИТИРОВАННОЙ ЛИТЕРАТУРЫ}

[1] Аграчев А. А. Топология квадратичных отображений и гессианы гладких отображений // Итоги науки и техники. Алгебра. Геометрия. Топология. Т. 26. М.: ВИНИТИ, 1988. C. $85-124$.

[2] Dines L. L. On the mapping of quadratic forms // Bull. Amer. Math. Soc. 1941. V. 47. P. 494-498.

[3] Арутюнов А. В. Условия экстремума. Анормальные и вырожденные задачи. М.: Факториал, 1997.

[4] Polyak B.T. Convexity of Quadratic Transformations and Its Use in Control and Optimization // J. Optimiz. Theory Appl. 1998. V. 99. № 3.

[5] Арутюнов А. В., Ячимович В. К теории экстремума для анормальньх задач // Вестн. МГУ. Сер. 15. Выч. матем., киберн. 1999. № 4. С. 34-40.

[6] Аваков Е. Р., Аграчев А. А., Арутюнов А. В. Множество уровня гладкого отображения в окрестности особой точки и нули квадратичного отображения // Матем. сб. 1991. Т. 182. № 8 . С. 1091-1104.

[7] Левитин Е.С., Милютин А.А., Осмоловский Н. П. Условия высших порядков локального минимума в задачах с ограничениями // УМН. 1978. Т. 33. №6. С. 85-148.

Российский университет дружбы народов, г. Москва

Поступило 30.08 .2000 